\title{
Correction to: Rare case of an upper urinary tract carcinoma (UTUC) in renal pelvis and ureter associated to renal vein thrombosis: diagnostic imaging with CECT, MRI and CEUS
}

\author{
Matteo Mastrorosato ${ }^{1}$. Elena Bertelli ${ }^{1} \cdot$ Maria Cristina Bonini ${ }^{1} \cdot$ Ginevra Danti ${ }^{1}\left[{ }^{10} \cdot\right.$ Costanza Vannini $^{1}$. \\ Simone Agostini ${ }^{1} \cdot$ Vittorio Miele $^{1}$
}

Published online: 18 July 2019

(c) Società Italiana di Ultrasonologia in Medicina e Biologia (SIUMB) 2019

\section{Correction to: Journal of Ultrasound https://doi.org/10.1007/s40477-019-00396-z}

Unfortunately, the first name and family names of all the authors have incorrectly been swapped and published in original article.

The complete correct names of all the authors are given below:

Matteo Mastrorosato, Elena Bertelli, Maria Cristina Bonini, Ginevra Danti, Costanza Vannini, Simone Agostini, Vittorio Miele.

The original article can be found online at https://doi.org/10.1007/ s40477-019-00396-z.

Ginevra Danti

ginevra.danti@gmail.com

Matteo Mastrorosato

matteo.mastrorosato@gmail.com

Elena Bertelli

elena.bertelli3@gmail.com

Maria Cristina Bonini

boninimariacristina@gmail.com

Costanza Vannini

costanzavannini@gmail.com

Simone Agostini

agostini.s@alice.it

Vittorio Miele

vmiele@ sirm.org

1 Department of Radiology, Azienda Ospedaliero-

Universitaria Careggi, Largo G.A. Brambilla 3,

50134 Florence, Italy 